\title{
Surgery of Colorectal Liver Metastases: Pushing the Limits
}

\author{
Guido Torzillia René Adam ${ }^{b} \quad$ Luca Viganò $^{a}$ Katsunori Imaib \\ Jeremias Goranskyc Andrea Fontana ${ }^{\mathrm{a}}$ Christian Toso ${ }^{\mathrm{d}}$ \\ Pietro Majnod Eduardo de Santibañes ${ }^{c}$ \\ aDepartment of Surgery, Division of Hepatobiliary and General Surgery, Humanitas University, Hu- \\ manitas Clinical and Research Hospital, Rozzano, Italy, ${ }^{b}$ Centre Hépato-Biliaire, AP-HP, Hôpital Uni- \\ versitaire Paul Brousse, Villejuif, France, 'Department of General Surgery, Division of Hepatobiliary \\ and Pancreatic Surgery, Hospital Italiano de Buenos Aires, Buenos Aires, Argentina, dDepartment of \\ Visceral and Transplantation Surgery, University Hospitals of Geneva, Geneva, Switzerland
}

\section{Key Words}

Complications · Metastasis · Outcome $\cdot$ Surgery $\cdot$ Systemic chemotherapy

\begin{abstract}
Background: The recent advent of more effective chemotherapy and the development of surgical procedures have expanded the pool of resectable patients with colorectal liver metastases (CLM). Two-stage hepatectomy (TSH), associated liver partition and portal vein ligation for staged hepatectomy (ALPPS), and ultrasound-guided enhanced one-stage hepatectomy (e-OSH) are the surgical solutions proposed for these patients, but the range of indications for these procedures vary from institution to institution. Summary: The advantages and disadvantages of each approach are herein discussed. Patients who drop out between the staged operations of TSH limit its success rate, although predictive scores may help with patient selection and thereby optimize the results. Safety and oncological suitability are concerns to be addressed when considering ALPPS. These concerns notwithstanding, ALPPS has introduced an innovative concept in surgery: the monosegmental remnant liver. Studies involving e-OSH have proven the oncological suitability of tumor exposure once the CLM is detached from major intrahepatic vessels. This finding could expand the indications for e-OSH, although the technical challenges that it entails limit its spread among the surgical community. The liver-first approach involves the clearance of tumors from the liver before the colorectal primary is tackled. This approach fully justifies the complexity of e-OSH. Key Messages: Predictive scores limiting the interstage dropout of TSH, partial and monosegmental ALPPS, and R1 vascular e-OSH justified by solid long-term results represent new
\end{abstract}


insights that could help refine the patient assignment to each of these approaches. Additionally, liver transplantation is an emerging treatment for CLM that should be taken into account.

Copyright $(2016$ S. Karger AG, Basel

\section{Introduction}

The recent advent of more effective chemotherapy and the development of surgical procedures have expanded the pool of resectable patients with colorectal liver metastases (CLM). The treatment of patients with multiple bilobar nodules is particularly complex because a large amount of parenchyma often must be sacrificed, which leads to the risk of postoperative liver failure [1]. To prevent this risk, in 2000, two-stage hepatectomy (TSH) was proposed [2]. TSH is now a standardized procedure adopted worldwide with good shortand long-term results [1,3-10]: its major drawback is the dropout risk as a result of disease progression between the two stages [11-12].

To overcome this dropout risk, some alternatives have been advanced, e.g., associating liver partition and portal vein ligation for staged hepatectomy (ALPPS) $[13,14]$ and ultrasound-guided enhanced one-stage hepatectomy (e-OSH) [15]. ALPPS reduces the number of dropouts but is still associated with elevated postoperative mortality. Ultrasound-guided e-OSH is a parenchyma-sparing approach even for deep-located lesions, but it involves a level of complexity that many surgical teams consider unsuitable, even in specialized centers. These approaches are discussed in this article.

\section{Two-Stage Hepatectomy}

Indications and Methods

Indications are:

1. Bilobar CLM

2. Largest CLM $>30 \mathrm{~mm}$ and/or $>3$ CLM in the future remnant liver (FRL) and FRL $>30 \%$ of the total liver volume.

This strategy aims to achieve liver tumor clearance sequentially:

Stage 1: Clearance of tumor from the less invaded liver (usually the left), and portal vein ligation (PVL) combined with portal vein embolization (PVE) using dehydrated ethanol to induce FRL hypertrophy $[1,2,12]$ (fig. 1).

Stage 2: Lobectomy or extended lobectomy once the FRL is considered to be large enough (FRL $>30 \%$ and/or FRL/total body weight ratio $>0.5 \%$ ).

If the primary tumor is synchronously presented, its resection is performed at the same time as the first-stage hepatectomy; otherwise it is resected after the second-stage hepatectomy. A recent review reported that simultaneous resection of the primary tumor was performed at first-stage hepatectomy in a median of $30 \%$ of patients [3]. Previous studies have reported that simultaneous resection of the primary tumor at first-stage hepatectomy did not affect the postoperative course $[16,17]$ and allowed optimization of chemotherapy administration [16]. From this perspective, optimal first-line chemotherapy should be a short course to minimize dropout and liver injury [12].

Interval chemotherapy is generally recommended 3 weeks after first-stage hepatectomy using the same regimen as that used preoperatively or, in some centers, chemotherapy is adopted selectively in cases with disease progression between stages [6]. However, in two studies, interval chemotherapy failed to reduce the dropout rate $[11,12]$. Consequently, its 


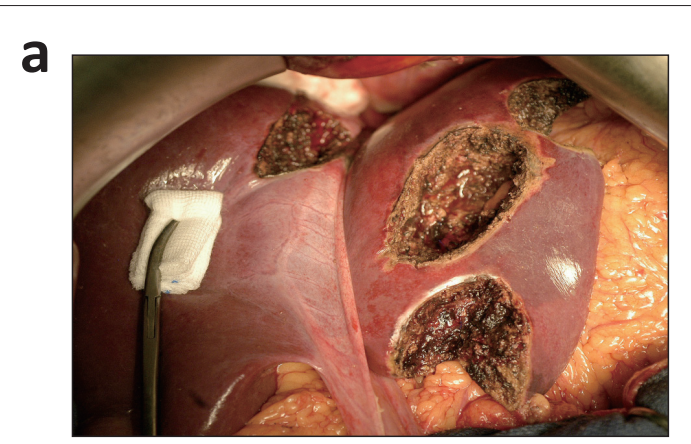

Clearance of left hemiliver

C

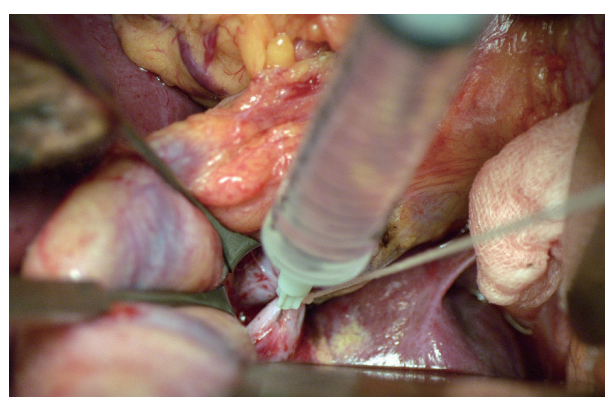

Embolization using dehydrated ethanol b

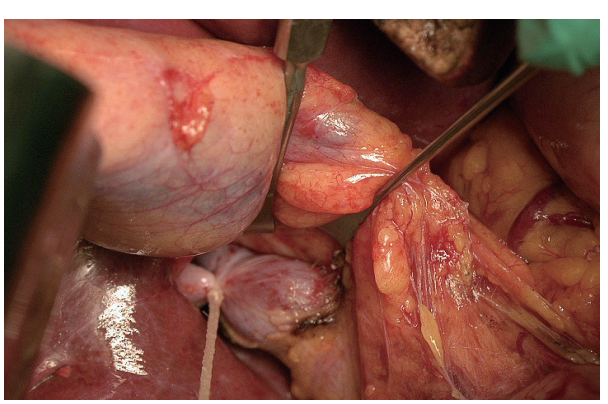

Ligation of Rt. Portal vein

Fig. 1. The TSH procedure. a During first-stage hepatectomy, in most cases, the less-invaded liver lobe is cleared of its metastases, usually by non-anatomical resection. b Ligation of the right portal vein. c Embolization by dehydrated ethanol. To improve the safety of second-stage hepatectomy, PVL and embolization is routinely performed during first-stage hepatectomy. Rt.=right.

effectiveness deserves to be further investigated. Similarly, despite initial positive experiences [1], the role of postoperative chemotherapy is not universally accepted as beneficial.

\section{Results}

Ninety-day mortality rates were $0.8 \%$ and $2.5 \%$ after first-stage and second-stage hepatectomy, respectively ( $\mathrm{p}=0.97$ ), and postoperative complication rates (Clavien $\geq$ III [18]) were $14 \%$ and $33 \%$, respectively ( $\mathrm{p}=0.0015$ ) [12]. Previously reported 5-year overall survival (OS) rates after completion of TSH ranged from $32 \%$ to $64 \%$, with median survival times of 24-44 months $[1,3-12,19,20]$. Preoperative chemotherapy cycles $\geq 6$ [10], tumor number $\geq 6$ [1], the presence of concomitant extrahepatic disease [1], and no postoperative chemotherapy [1] were reported to be independent prognostic factors of poor survival after completion of TSH. However, the main drawback of TSH is patient dropout, which mainly results from disease progression between the two stages (56-100\%, median, 100\%) [3].

The most recent data at Paul Brousse Hospital (PBH) indicate a failure rate of 35\% [12] and an OS for patients who failed TSH significantly lower than that for those who completed TSH (1-, 3-, 5-year OS rates: 66\%, $14 \%$ and $0 \%$ vs. 95\%, 69\%, and $44 \%, \mathrm{p}<0.0001$ ) [12].

Some independent predictive factors of dropout have been proposed: CLM number in the FRL $\geq 3$ [19], age $\geq 70$ years [19], combined resection of primary tumor [20], and disease progression during chemotherapy [10]. Recently, a predictive score was proposed that includes preoperative carcinoembryonic antigen $>30 \mathrm{ng} / \mathrm{ml}$, tumor size $>40 \mathrm{~mm}$, preoperative chemotherapy cycles $>12$, and disease progression during first-line chemotherapy [12]. Patients presenting with none of these factors had a probability of TSH failure of $10.5 \%$, and the addi- 
tion of each subsequent factor increased the risk to $43 \%, 73 \%, 88 \%$, and $95 \%$. This model may contribute to a better selection of patients who are suitable for TSH.

\section{ALPPS}

ALPPS is a short-term TSH that induces an unparalleled increase in FRL volume [21]. It has the following advantages:

- Unparalleled rapid liver hypertrophy [13,14,22]: FRL of just one segment [23]

- Rescue from PVE or PVL failure [24]

- Shortening of the interval between the two stages, thereby minimizing the dropout risk and adhesion formation [14,21-23,25,26].

- Maximizing the rate of R0 resections to approaching 100\% [22,27].

Indications and Technical Pointers

Indications can be summarized as follows [25]:

1. Unilateral single or multiple lesions in close contact to the FRL or its vascular pedicles

2. Bilateral disease

3. Failure of PVE/PVL

4. Unexpected tumor extension during surgical exploration

5. Large hypertrophy needed.

Patients younger than 60 years with CLM $[22,26]$ benefit most from this approach.

The strategy aims to achieve liver tumor clearance sequentially but is faster overall than TSH:

Stage 1: Clearance of the FRL is performed together with PVL and liver parenchyma division Stage 2: Lobectomy or extended lobectomy is performed when the FRL is considered to be large enough (about 10 days after stage 1) [25].

\section{Results}

Preliminary experiences with ALPPS were associated with high morbidity and mortality rates $[13,28]$. However, when analyzing the most recent data that compares the results of TSH from the largest published series versus those of ALPPS in the worldwide registry and in the largest single center experiences, it becomes evident that there is not really a difference between these two approaches in terms of major morbidity, liver failure, or mortality rates [29]. Recent data from the ALPPS worldwide registry showed a major morbidity rate of $36 \%$ and a mortality rate of $7.5 \%$ [22]. In contrast, major morbidity rates of $59 \%$ and $40 \%$, liver failure rates of $22 \%$, and mortality rates of $10 \%$ and $3 \%$ were published for TSH in a $\mathrm{PBH}$ series and in a systematic review of TSH, respectively $[1,29]$.

Age, the presence of biliary tumors, operative times more than $5 \mathrm{~h}$ during stage 1 , and the administration of red blood cell transfusions during either stage have been identified as significant risk factors for severe complications and 90-day mortality. In addition, recent data from the ALPPS registry indicated that patients who develop post-hepatectomy liver failure after stage 1 or have a Model for End-stage Liver Disease score $\geq 10$ before stage 2 are at higher risk, with adjusted odds of 90-day mortality of 3.9 [confidence interval (CI) 1.4-10.9] and 4.9 (CI 1.9-12.7), respectively. In a recently published single series, only total liver partition was associated with an increased risk of morbidity under both univariate and multivariate analysis. Interestingly, partial parenchymal transection has been associated with lower morbidity and equal hypertrophic results [25]. 
In terms of oncological outcomes, because this is a new procedure, only short-term follow up data are available for analysis. The OS at 1 and 2 years was $59 \%$ and $41 \%$, and the diseasefree survival at 1 and 2 years was $88 \%$ and $74 \%$, respectively, from the ALPPS registry. These findings compare favorably with those provided in the few existing international series of $\mathrm{TSH}$, in which patients who did not reach the second stage were excluded from the survival analysis in most cases $[1,4,22,29,30]$. When comparing resectability between ALPPS and TSH, even though no randomized studies have been performed, there seems to be evidence in favor of ALPPS. A multicenter study compared the rate of complete resection between ALPPS and TSH in patients with oncologically unfavorable factors. This study showed that the rate of complete resection in ALPPS was greater than that in TSH, with 83\% in ALPPS vs 63\% in TSH $(\mathrm{p}<0.001$ ) [27]. Moreover, the resectability rate published in the ALPPS registry was $98 \%$ [22], whereas that published for TSH in a systematic review was 76\% [29].

\section{One-Stage Hepatectomy}

An alternative approach for multiple bilobar CLM is OSH, i.e., the resection is carried out in a single procedure for all nodules. OSH is commonly adopted for superficial lesions (cherrypicking surgery) or, less commonly, for more complex procedures such as right hepatectomy and left liver parenchymal-sparing tumor clearance after PVE [31]. Torzilli et al. extended the parenchymal sparing approach to deep-located CLM (including those in contact with major intrahepatic vessels) and introduced the concept of e-OSH [15,32], thereby limiting the use of PVE to exceptional conditions [33] (fig. 2).

\section{Indications and Technical Pointers}

e-OSH relies on:

1. The use of intraoperative ultrasonography (IOUS) to guide resection [34]

2 . The detachment of CLM from intrahepatic vascular structures $[35,36]$. The equivalence between CLM-vessel detachment R1 vascular margin (R1Vasc) and R0 surgery has been recently demonstrated [37]. Portal pedicles are resected only if infiltrated or if upstream biliary dilatation is evident. Hepatic veins (HV) are spared or are tangentially resected and reconstructed (direct suture or patch), except in patients with thrombosis or CLM surrounding $>2 / 3$ of the HV circumference

3. An accurate flow analysis identifying communicating veins among HVs to spare parenchyma even after HV section [38].

\section{Results}

These e-OSH techniques minimize the need for major hepatectomies and two-stage resections. In the Humanitas Research Hospital, as of June 2015, among 164 patients with illlocated multiple bilobar CLM, only 9 needed to undergo TSH. As recently reported, among the other 155 patients who underwent e-OSH for a median of 7 (range, 4-49) CLM removed per patient, 63 (41\%) had more than $10 \mathrm{CLM}$. All patients had at least one contact between a CLM and a major intrahepatic vessel (first/second-order Glissonian pedicles and/or HV at the caval confluence), and 55 (35\%) patients had contact bilaterally: mortality and major morbidity was $1.2 \%$ and $7 \%$, respectively, with a 5 -year OS of $32 \%$ [32]. The nine patients who underwent TSH had CLM surrounding $360^{\circ}$ of the first/second-order Glissonian bifurcation (providing no room for any partial sparing of the right liver) and would have had an inadequate FRL. 


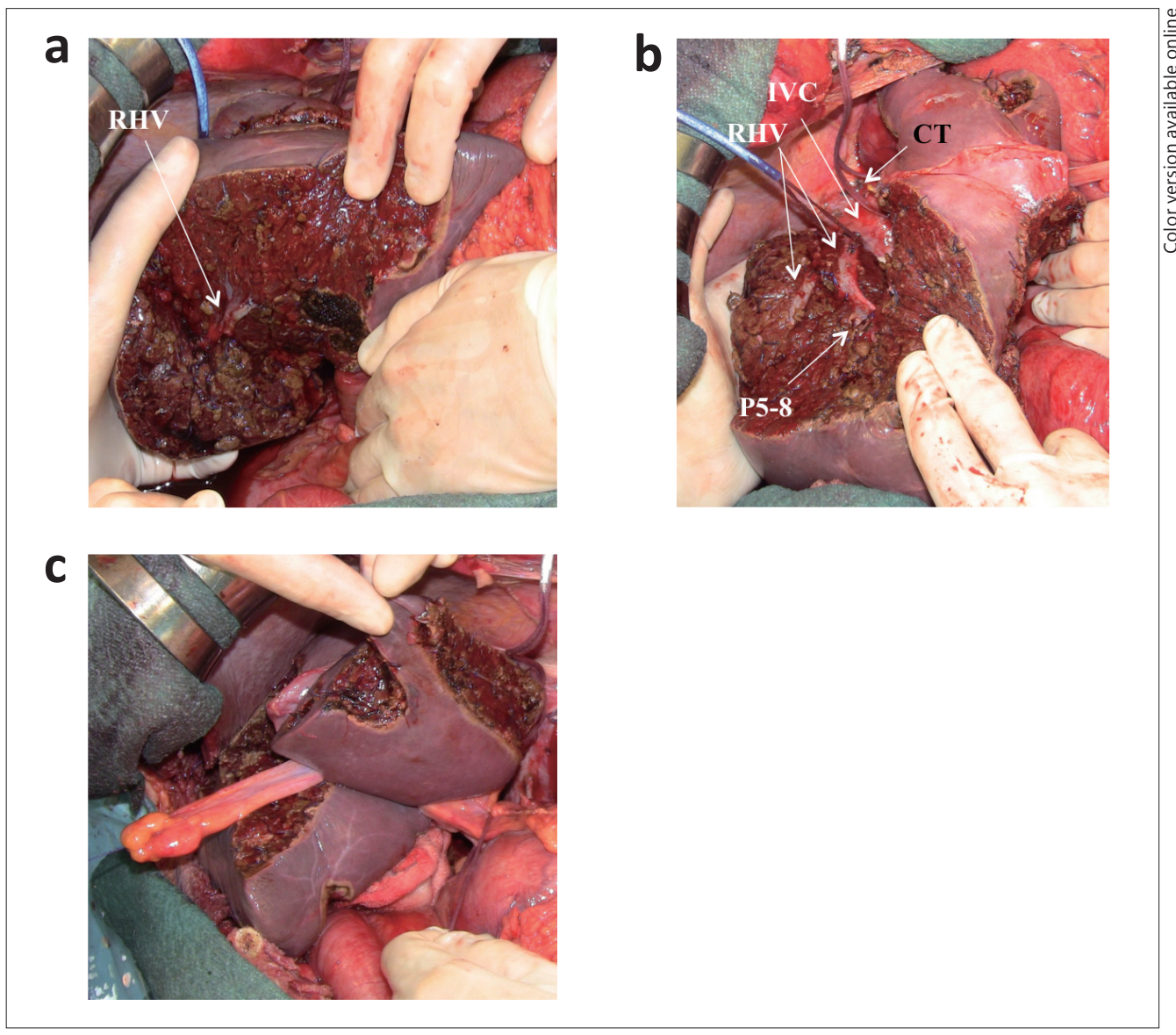

Fig. 2. A patient with 44 CLM underwent e-OSH. a Posterior surface of the right hepatic vein (RHV) at the mid portion; (b) the tunnel with the RHV and the dorsal surface of the right anterior portal branch (P5-8) exposed; (c) the left-side resected areas. IVC=Inferior vena cava; CT=common trunk.

Recently, a case-matched analysis was carried out of patients with similar tumor burdens ( $\geq 6$ CLM, at least one major vascular contact, and more than three CLM larger than $3 \mathrm{~cm}$ in the less involved hemiliver) that compared the outcomes of patients who underwent e-OSH at the Humanitas Research Hospital and TSH at the PBH. Compared with those undergoing TSH, patients undergoing e-OSH had lower blood loss (500 vs. $1100 \mathrm{ml}, \mathrm{p}=0.009$ ), lower severe morbidity ( $14 \%$ vs. $36 \%, \mathrm{p}=0.04$ ), and lower liver-specific morbidity ( $23 \%$ vs. $41 \%, \mathrm{p}=0.02$ ). The $\mathrm{R} 0$ resection rate was similar (17\% vs. $11 \%$, TSH dropout $41 \%$ ). e-OSH and completed TSH had similar OS rates. Regarding recurrence sites and recurrence-free survival, a recent report indicated that they were similar for e-OSH and TSH [39], calling into question the supposed higher risk of recurrence in the remnant liver after parenchymasparing surgery compared to that in major hepatectomies.

In a recent report analyzing a prospective cohort of 226 patients undergoing liver resection for CLM (627 resection areas), local recurrence risk was similar between the R0 and R1Vasc groups (per-patient analysis 5.3\% vs. 4.3\%; per-resection area analysis $1.5 \%$ vs. 3.9\%), but increased in the R1 parenchyma (R1Par) group (19.6\% and $13.6 \%, \mathrm{p}<0.05$ ), independent of chemotherapy [37]. R1Par was an independent negative prognostic factor of survival ( $\mathrm{p}=0.034)$; conversely R1Vasc versus R0 had no significant differences in OS. Therefore, R1Vasc achieves local disease control equivalent to R0 with equivalent survival. Consequently, CLM detachment from intrahepatic vessels is a suitable approach from the 
oncological standpoint; this evidence legitimizes the use of e-OSH for patients with profiles that probably overlap with those undergoing TSH or ALPPS.

\section{Liver-First Approach}

The aforementioned approaches are often offered to patients with synchronous bowel disease that responded to systemic therapy, which means that the primaries are generally asymptomatic and at low risk of causing occlusion. The priority, from a prognostic standpoint, is to address the liver involvement. A liver-first approach [40] is generally preferred in the case of $\mathrm{OSH}$, whereas synchronous resection of the primary and tumor clearance of the less involved liver is often selected in staged procedures.

Mentha et al. introduced the liver-first policy in a pilot study with few patients who underwent a variety of chemotherapy treatments $[40,41]$. More recently, we carried out a retrospective analysis of 49 patients treated in Geneva, all of whom underwent oxaliplatin-based chemotherapy (and some also received bevacizumab). The results obtained were satisfactory in terms of OS: after a median follow-up of 33 months, 3- and 5-year OS were $64 \%$ and 55\%, respectively, with a median progression-free survival of 14 months. Mortality was zero and major morbidity occurred in $6 \%$ of patients. The primary tumors could be resected in all patients. Colorectal recurrences have been observed in five patients so far, all with rectal tumors. These results emphasize the efficacy, safety, and tolerance of this strategy and have relevance for those patients indicated as suitable for e-OSH, TSH, or ALPPS.

\section{Conclusions}

In the absence of a definite consensus, the ranges of indications for TSH, ALPPS, and eOSH vary among different institutions. Debating and comparing patient profiles and results is the only way a consensus on specific indications can be achieved. However, it may generally be accepted that, whenever feasible, e-OSH should, theoretically at least, be the preferred approach because it entails a single surgical session with no dropout and avoids the increased risk of double (or triple in case of PVE) interventions.

Approaching even deep-located CLM conservatively by applying the concept of R1vasc resection (now supported by a consistent oncological background [37]) should reinforce the role played by e-OSH for such patients, thereby limiting the need for staged procedures.

However, e-OSH may not always be feasible, in which case staged procedures play their part. Certainly, for TSH, the heterogeneity of results in terms of dropouts is a concern, as the safety and oncological suitability are for ALPPS. Both TSH and ALPPS suffer from the lack of established indications, resulting in a wide variety of different liver involvement in those undergoing the procedures. Additionally, ALPPS has a relatively short history.

Suitable predictive scores may help improve patient selection for TSH [12], thereby reducing the risk of dropout. ALPPS is still very new; oncological outcomes remain uncertain and mortality is still high. However, rapid progress along the learning curve (with recent reports of series with no mortalities), the introduction of some adjustments (e.g., partial ALPPS [25]), and the perspectives opened by monosegmental ALPPS [23] presage an interesting future for this technique.

TSH and ALPPS could become complementary approaches to the treatment of CLM, with ALPPS reserved for scenarios with a very small FRL or as rescue of failed PVE. In contrast, the application of e-OSH depends essentially on the diffusion of a policy, in that it requires not 
only technical skill in IOUS-guided surgery but also the assimilation of surgical techniques considered controversial by many surgeons, mostly in western countries, such as the thoraco-abdominal approach [42].

The liver-first approach, i.e., prioritizing the prognostic impact in stage IV colorectal cancer of the metastatic involvement of the liver, represents a chance to achieve complete tumor clearance and can be accomplished by either single-session or staged operations. In particular for the e-OSH, it is an almost obliged policy, being too risky the association of a complex liver resections with the colorectal surgery. Therefore, e-OSH becomes, in effect, a staged surgery when synchronous disease exists. On the other hand, in staged procedures, FRL tumor clearance at the first stage is frequently associated with removal of the primary tumor [3], limiting de facto the need for a liver-first approach. The difference between a "staged" e-OSH and staged operations with associated primary tumor removal at the first stage of a two-stage procedure could be that, because the liver is the organ that in most cases determines surgical feasibility, dropout is less frequent in single-stage liver clearance compared with that in two-stage procedures.

A final remark should be made concerning liver transplantation, which is progressively being carried out in CLM patients [43]: this approach could, for selected patients, obviate the need for the complex procedures herein discussed.

This review article gives an update on the different and sometimes competing approaches to the treatment of advanced CLM; it does not pretend to offer a comprehensive statement, which would in any case be premature at this stage. However, we have provided a complete overview that profits from contributions reflecting the diverse clinical practices of the authors. We are confident that this article will give the reader an insight into this complex and still unresolved issue.

\section{Conflict of Interest}

The authors state that no conflicts of interest exist.

\section{References}

1 Wicherts DA, Miller R, de Haas RJ, Bitsakou G, Vibert E, Veilhan LA, Azoulay D, Bismuth H, Castaing D, Adam R: Long-term results of two-stage hepatectomy for irresectable colorectal cancer liver metastases. Ann Surg 2008;248:994-1005.

2 Adam R, Laurent A, Azoulay D, Castaing D, Bismuth H: Two-stage hepatectomy: A planned strategy to treat irresectable liver tumors. Ann Surg 2000;232:777-785.

3 Chua TC, Liauw W, Chu F, Morris DL: Summary outcomes of two-stage resection for advanced colorectal liver metastases. J Surg Oncol 2013;107:211-216.

4 Tsai S, Marques HP, de Jong MC, Mira P, Ribeiro V, Choti MA, Schulick RD, Barroso E, Pawlik TM: Two-stage strategy for patients with extensive bilateral colorectal liver metastases. HPB Oxf 2010;12:262-269.

5 Tsim N, Healey AJ, Frampton AE, Habib NA, Bansi DS, Wasan H, Cleator SJ, Stebbing J, Lowdell CP, Jackson JE, Tait P, Jiao LR: Two-stage resection for bilobar colorectal liver metastases: R0 resection is the key. Ann Surg Oncol 2011;18:1939-1946.

6 Brouquet A, Abdalla EK, Kopetz S, Garrett CR, Overman MJ, Eng C, Andreou A, Loyer EM, Madoff DC, Curley SA, Vauthey JN: High survival rate after two-stage resection of advanced colorectal liver metastases: response-based selection and complete resection define outcome. J Clin Oncol 2011;29:1083-1090.

7 Pamecha V, Nedjat-Shokouhi B, Gurusamy K, Glantzounis GK, Sharma D, Davidson BR: Prospective evaluation of two-stage hepatectomy combined with selective portal vein embolisation and systemic chemotherapy for patients with unresectable bilobar colorectal liver metastases. Dig Surg 2008;25:387-393.

8 Cardona K, Donataccio D, Kingham TP, Allen PJ, DeMatteo RP, Fong Y, Jarnagin WR, Cercek A, Kemeny NE, D'Angelica MI: Treatment of extensive metastatic colorectal cancer to the liver with systemic and hepatic arterial infusion chemotherapy and two-stage hepatic resection: the role of salvage therapy for recurrent disease. Ann Surg Oncol 2014;21:815-821. 
9 Brudvik KW, Seeberg LT, Hugenschmidt H, Renolen A, Schirmer CB, Brunborg C, Bjørnbeth BA, Borgen E, Naume B, Waage A, Wiedswang G: Detection of circulating tumor cells at surgery and at follow-up assessment to predict survival after two-stage liver resection of colorectal liver metastases. Ann Surg Oncol 2015;22:4029-4037.

10 Giuliante F, Ardito F, Ferrero A, Aldrighetti L, Ercolani G, Grande G, Ratti F, Giovannini I, Federico B, Pinna AD, Capussotti L, Nuzzo G: Tumor progression during preoperative chemotherapy predicts failure to complete 2-stage hepatectomy for colorectal liver metastases: results of an Italian multicenter analysis of 130 patients. J Am Coll Surg 2014;219:285-294.

11 Muratore A, Zimmitti G, Ribero D, Mellano A, Viganò L, Capussotti L: Chemotherapy between the first and second stages of a two-stage hepatectomy for colorectal liver metastases: should we routinely recommend it? Ann Surg Oncol 2012;19:1310-1315.

12 Imai K, Benitez CC, Allard MA, Vibert E, Cunha AS, Cherqui D, Castaing D, Bismuth H, Baba H, Adam R: Failure to achieve a 2-stage hepatectomy for colorectal liver metastases: how to prevent it? Ann Surg 2015;262:772-778, discussion 778-779.

13 Schnitzbauer AA, Lang SA, Goessmann H, Nadalin S, Baumgart J, Farkas SA, Fichtner-Feigl S, Lorf T, Goralcyk A, Hörbelt R, Kroemer A, Loss M, Rümmele P, Scherer MN, Padberg W, Königsrainer A, Lang H, Obed A, Schlitt HJ: Right portal vein ligation combined with in situ splitting induces rapid left lateral liver lobe hypertrophy enabling 2-staged extended right hepatic resection in small-for-size settings. Ann Surg 2012;255:405-414.

14 de Santibañes E, Clavien PA: Playing Play-Doh to prevent postoperative liver failure: the "ALPPS" approach. Ann Surg 2012;255:415-417.

15 Torzilli G, Procopio F, Botea F, Marconi M, Del Fabbro D, Donadon M, Palmisano A, Spinelli A, Montorsi M: One-stage ultrasonographically guided hepatectomy for multiple bilobar colorectal metastases: a feasible and effective alternative to the 2-stage approach. Surgery 2009;146:60-71.

16 Karoui M, Vigano L, Goyer P, Ferrero A, Luciani A, Aglietta M, Delbaldo C, Cirillo S, Capussotti L, Cherqui D: Combined first-stage hepatectomy and colorectal resection in a two-stage hepatectomy strategy for bilobar synchronous liver metastases. Br J Surg 2010;97:1354-1362.

17 Stella M, Dupre A, Chabaud S, Gandini A, Meeus P, Peyrat P, Rivoire M: A comparative study of patients with and without associated digestive surgery in a two-stage hepatectomy setting. Langenbecks Arch Surg 2012;397:1289-1296.

18 Dindo D, Demartines N, Clavien PA: Classification of surgical complications: a new proposal with evaluation in a cohort of 6336 patients and results of a survey. Ann Surg 2004;240:205-213.

19 Narita M, Oussoultzoglou E, Jaeck D, Fuchschuber P, Rosso E, Pessaux P, Marzano E, Bachellier P: Two-stage hepatectomy for multiple bilobar colorectal liver metastases. Br J Surg 2011;98:1463-1475.

20 Turrini O, Ewald J, Viret F, Sarran A, Goncalves A, Delpero JR: Two-stage hepatectomy: who will not jump over the second hurdle? Eur J Surg Oncol 2012;38:266-273.

21 de Santibañes E, Ardiles V, Alvarez FA: Associating liver partition and portal vein ligation for staged hepatectomy: a better approach to treat patients with extensive liver disease. JAMA Surg 2015;150:929-930.

22 Schadde E, Ardiles V, Robles-Campos R, Malago M, Machado M, Hernandez-Alejandro R, Soubrane O, Schnitzbauer AA, Raptis D, Tschuor C, Petrowsky H, De Santibanes E, Clavien PA, ALPPS Registry Group: Early survival and safety of ALPPS: first report of the International ALPPS Registry. Ann Surg 2014;260:829-836, discussion 836-838.

23 Schadde E, Malagó M, Hernandez-Alejandro R, Li J, Abdalla E, Ardiles V, Lurje G, Vyas S, Machado MA, de Santibañes E: Monosegment ALPPS hepatectomy: extending resectability by rapid hypertrophy. Surgery 2015;157:676-689.

24 Tschuor C, Croome KP, Sergeant G, Cano V, Schadde E, Ardiles V, Slankamenac K, Clariá RS, de Santibaňes E, Hernandez-Alejandro R, Clavien PA: Salvage parenchymal liver transection for patients with insufficient volume increase after portal vein occlusion - an extension of the ALPPS approach. Eur J Surg Oncol 2013;39:1230-1235.

25 Alvarez FA, Ardiles V, de Santibañes M, Pekolj J, de Santibañes E: Associating liver partition and portal vein ligation for staged hepatectomy offers high oncological feasibility with adequate patient safety: a prospective study at a single center. Ann Surg 2015;261:723-732.

26 Hernandez-Alejandro R, Bertens KA, Pineda-Solis K, Croome KP: Can we improve the morbidity and mortality associated with the associating liver partition with portal vein ligation for staged hepatectomy (ALPPS) procedure in the management of colorectal liver metastases? Surgery 2015;157:194-201.

27 Schadde E, Ardiles V, Slankamenac K, Tschuor C, Sergeant G, Amacker N, Baumgart J, Croome K, HernandezAlejandro R, Lang H, de Santibaňes E, Clavien PA: ALPPS offers a better chance of complete resection in patients with primarily unresectable liver tumors compared with conventional-staged hepatectomies: results of a multicenter analysis. World J Surg 2014;38:1510-1519.

28 Torres OJ, Fernandes ES, Oliveira CV, Lima CX, Waechter FL, Moraes-Junior JM, Linhares MM, Pinto RD, Herman P, Machado MA: Associating liver partition and portal vein ligation for staged hepatectomy (ALPPS): the Brazilian experience. Arq Bras Cir Dig 2013;26:40-43.

29 Lam VW, Laurence JM, Johnston E, Hollands MJ, Pleass HC, Richardson AJ: A systematic review of two-stage hepatectomy in patients with initially unresectable colorectal liver metastases. HPB Oxf 2013;15:483-491.

30 Abbott DE, Sohn VY, Hanseman D, Curley SA: Cost-effectiveness of simultaneous resection and RFA versus 2-stage hepatectomy for bilobar colorectal liver metastases. J Surg Oncol 2014;109:516-520.

31 Kawasaki S, Makuuchi M, Kakazu T, Miyagawa S, Takayama T, Kosuge T, Sugihara K, Moriya Y: Resection for multiple metastatic liver tumors after portal embolization. Surgery 1994;115:674-677. 
32 Torzilli G, Cimino M: Extending the Limits of Resection for Colorectal Liver Metastases ENHANCED ONE STAGE SURGERY. J Gastroint Surg, 2016; 10.1007/s11605-016-3250-8.

33 Torzilli G, Donadon M, Palmisano A, Marconi M, Procopio F, Botea F, Del Fabbro D, Cappellani A, Montorsi M: Ultrasound guided liver resection: does this approach limit the need for portal vein embolization? Hepatogastroenterol 2009;56:1486-1490.

34 Torzilli G: Ultrasound-guided liver surgery. An atlas. ed 1. Springer, 2014.

35 Torzilli G, Montorsi M, Del Fabbro D, Palmisano A, Donadon M, Makuuchi M: Ultrasonographically guided surgical approach to liver tumours involving the hepatic veins close to the caval confluence. Br J Surg 2006;93:1238-1246.

36 Torzilli G, Procopio F, Costa G: Adjuncts to hepatic resection - ultrasound and emerging guidance systems; in: Jarnagin WR, Belghiti J, Blumgart LH: Blumgart's Surgery of the Liver, Pancreas, and Biliary Tract. Edn 5, Philadelphia, Elsevier Saunders. 2012, vol. 2, pp 1604-1649.

37 Viganò L, Procopio F, Cimino MM, Donadon M, Gatti A, Costa G, Del Fabbro D, Torzilli G: Is tumor detachment from vascular structures equivalent to r0 resection in surgery for colorectal liver metastases? An observational cohort. Ann Surg Oncol 2016;23:1352-1360.

38 Torzilli G, Garancini M, Donadon M, Cimino M, Procopio F, Montorsi M: Intraoperative ultrasonographic detection of communicating veins between adjacent hepatic veins during hepatectomy for tumours at the hepatocaval confluence. Br J Surg 2010;97:1867-1873.

39 Viganò L, Torzilli G, Cimino M, Imai K, Vibert E, Donadon M, Castaing D, Adam R: Drop-out between the two liver resections of two-stage hepatectomy. Patient selection or loss of chance? Eur J Surg Oncol 2016;42:1385-1393.

40 Mentha G, Majno PE, Andres A, Rubbia-Brandt L, Morel P, Roth AD: Neoadjuvant chemotherapy and resection of advanced synchronous liver metastases before treatment of the colorectal primary. Br J Surg 2006;93:872-878.

41 Buchs NC, Ris F, Majno PE, Andres A, Cacheux W, Gervaz P, Roth AD, Terraz S, Rubbia-Brandt L, Morel P, Mentha G, Toso C: Rectal outcomes after a liver-first treatment of patients with stage IV rectal cancer. Ann Surg Oncol 2015;22:931-937.

42 Donadon M, Costa G, Gatti A, Torzilli G: Thoracoabdominal approach in liver surgery: how, when, and why. Updates Surg 2014;66:121-125.

43 Hagness M, Foss A, Line PD, Scholz T, Jørgensen PF, Fosby B, Boberg KM, Mathisen O, Gladhaug IP, Egge TS, Solberg S, Hausken J, Dueland S: Liver transplantation for nonresectable liver metastases from colorectal cancer. Ann Surg 2013;257:800-806. 